\title{
Where is my pain?
}

Pain is more than an unpleasant feeling associated with a somatosensory sensation. It is an important signal that prompts identification, localization, and reaction to a potential physical threat for the body. Detection and localization of a nociceptive stimulus and the ensuing percept of pain are largely determined by attention. For example, it has been shown that covert movement of spatial attention on the body can modify the whole cortical processing of nociceptive stimuli [9]. Even more striking evidence is provided in the present issue of PAIN ${ }^{\circledR}$ by Liu et al. [11], who studied detection of nociceptive stimuli in braindamaged patients with a neglect syndrome. Neglect denotes the impaired ability to process or to respond to sensory stimuli presented in the hemiportion of space contralateral to the side of the lesion (most often in the right hemisphere) [4]. A well-known demonstration that neglect is an attentional dysfunction that can occur in the absence of clear sensory and motor deficits is the description of patients who failed to report locations on the left side of the Piazza del Duomo in Milano when they were asked to mentally describe the Piazza from a specific perspective [3]. When they were asked to imagine that they had moved to the opposite side of the Piazza, which rotated the perspective by $180^{\circ}$, they neglected the left side of the new perspective, including locations that were correctly reported in the previous perspective. Conversely, they described accurately locations on the right side, including those of the neglected side in the previous perspective. A similar attentional disorder is extinction, defined as an inability to report or to respond to stimuli presented in the space contralateral to the side of the lesion when the stimuli are delivered in competition with stimuli presented in the space ipsilateral to the side of the lesion [4]. The classic test of tactile extinction is a double stimulation task in which tactile stimulation of the 
contralesional hand is correctly reported by patients when the contralesional hand is stimulated in isolation, but the same stimulus is no longer detected when the ipsilesional hand is stimulated simultaneously.

Complementary to previous descriptions of hemianesthesia after right brain damage [14], Liu et al. [11] described two patients with nociceptive extinction. These patients were able to report correctly the occurrence of a nociceptive stimulus when it was applied to their contralesional hand alone. However, the same stimulus was extinguished when it was delivered concomitantly with a nociceptive stimulus delivered to the ipsilesional hand. Liu et al. [11] described also seven neglect patients with nociceptive allesthesia: the patients identified correctly the occurrence of a nociceptive stimulus applied to the contralesional hand, but they localized the stimulus as if it had been applied to the ipsilesional hand. These observations demonstrate that in some circumstances the attentional weight that is given to a nociceptive stimulus can be dramatically changed so that its perception becomes completely distorted.

An important question that remains to be addressed is which maps are used by attention to focus on selective parts of the body and to localize a nociceptive stimulus. Most research on this topic has focused on the somatotopic organization of the cortical areas that receive nociceptive input $[1,2]$, based on the ordered projection from spatially organized receptive fields to spatially segregated subgroups of neurons in the cortex [6]. However, anatomical representations of the body alone can be insufficient to localize correctly the source of pain. For instance, when we are stung by a wasp on the left hand, an efficient action (e.g., chasing the wasp away) requests to shift the gaze and to move the right hand to the left space when the hands are in a normal posture, but to the right space when the hands are in a crossed posture, i.e., when the left hand resides in the right space. This was 
illustrated in a recent experiment by Moseley et al. [13], who investigated neglect-like behaviors in patients with complex regional pain syndrome (CRPS). During the concurrent stimulation with tactile stimuli applied both to the affected and the unaffected limbs, and when the limbs were in a normal posture, CRPS patients tended to neglect the stimulus applied to the affected limb, compared to the stimulus applied to the unaffected. In contrast, when the limbs were crossed, CRPS patients tended to neglect the stimulus applied to the unaffected limb compared to the stimulus applied to the affected limb. This observation clearly shows that the deficit observed in these patients is based on a spatial representation of the body which is independent of the somatotopic location of the symptoms.

If we take into account the data from studies that showed a close interaction between nociception and another sensory modality, such as vision [5,12], it can be concluded that the observations made by Liu et al. [11] and Moseley et al. [13] support the view that the detection and localization of a nociceptive stimulus strongly depends on the attention allocated to the part of the body on which the stimulus is applied, and that the frame of reference which is used to orient attention on the body is a representational frame of reference that depends on the posture of the body (i.e., the position of the limbs relative to each other), the sight of the limbs, and the integration of information from the different senses [10]. This extension of the body in external space, called the peripersonal space (Fig. 1), allows constructing a stable perception of external objects which is necessary to interact more dynamically and more efficiently with the environment [8]. The studies presented here, along with other studies, suggest that nociceptive inputs are integrated in a multimodal system that monitors the section of external space which constitutes a kind of margin of security against potential threats for the body [10]. The studies of Liu et al. [11] 
and Moseley et al. [13] open new perspectives in which a comprehensive functional significance of pain is considered: to signal potential physical threats so as to prompt defensive action.

\section{Conflict of interest}

The author has no conflict of interest related to this commentary.

\section{Acknowledgment}

The author is supported by the Research Foundation Flanders (FWO), Belgium.

\section{References}

[1] Baumgärtner U, lannetti GD, Zambreanu L, Stoeter P, Treede RD, Tracey I. Multiple somatotopic representations of heat and mechanical pain in the operculo-insular cortex: a high-resolution fMRI study. J Neurophysiol 2010;104:2863-72.

[2] Bingel U, Lorenz J, Glauche V, Knab R, Gläscher J, Weiller C, Büchel C. Somatotopic organization of human somatosensory cortices for pain: a single trial fMRI study. Neurolmage 2004;23:224-32.

[3] Bisiach E, Luzzatti C. Unilateral neglect of representational space. Cortex 1978;14:129-33.

[4] Heilman KM, Watson RW, Valenstein E. Neglect and related disorders. In: Heilman KM, Valenstein E, editors. Clinical neuropsychology. $4^{\text {th }}$ ed. Oxford: Oxford University Press, 2003; p 296-346.

[5] Hoogenraad TU, Ramos LMP, van Gijn J. Visually induced central pain and arm withdrawal after right parietal lobe infarction. J Neurol Neurosurg Psychiatr 1994;57:8502. 
[6] Kandel ER, Schwartz JH, Jessell TM. Principles of neural science. $4^{\text {th }}$ ed. New York: McGraw-Hill, 2000.

[7] Kerkhoff G. Spatial hemineglect in humans. Prog Neurobiol 2001;63:1-27.

[8] Làdavas E, Farnè A. Neuropsychological evidence for multimodal representations of space near specific body parts. In: Spence C, Driver J, editors. Crossmodal space and crossmodal attention. Oxford: Oxford University Press, 2004; p 69-98

[9] Legrain V, Guérit JM, Bruyer R, Plaghki L. Attentional modulation of the nociceptive processing into the human brain: selective spatial attention, probability of stimulus occurrence, and target detection effects on laser evoked potentials. Pain 2002;99:21-39.

[10] Legrain V, lannetti GD, Plaghki L, Mouraux A. The Pain Matrix reloaded. A saliencedetection system for the body. Prog Neurobiol (in press).

[11] Liu CC, Veldhuijzen DS, Ohara S, Winberry J, Greenspan JD, Lenz FA. Spatial attention to thermal pain stimuli in subjects with visual spatial hemi-neglect: extinction, mislocalization and misidentification of stimulus modality. Pain (in press).

[12] Longo MR, Betti V, Aglioti SM, Haggard P. Visually induced analgesia: seeing the body reduces pain. J Neurosci 2009:29;12125-30.

[13] Moseley GL, Gallace A, Spence C. Space-based, but not arm-based, shift in tactile processing in complex regional pain syndrome and its relationship to cooling of the affected limb. Brain 2009;132:3142-51.

[14] Sterzi R, Bottini G, Celani MG, Righetti E, Lamassa M, Ricci S, Vallar G. Hemianopsia, hemianaesthesia, anf hemiplegia after right and left hemisphere damage. A hemispheric difference. J Neurol Neurosurg Psychiatr 1993;56:308-10. 
Department of Experimental-Clinical and Health Psychology, Ghent University, Ghent, and Institute of Neuroscience, Université catholique de Louvain, Brussels, Belgium. E-mail address: valery.legrain@ugent.be

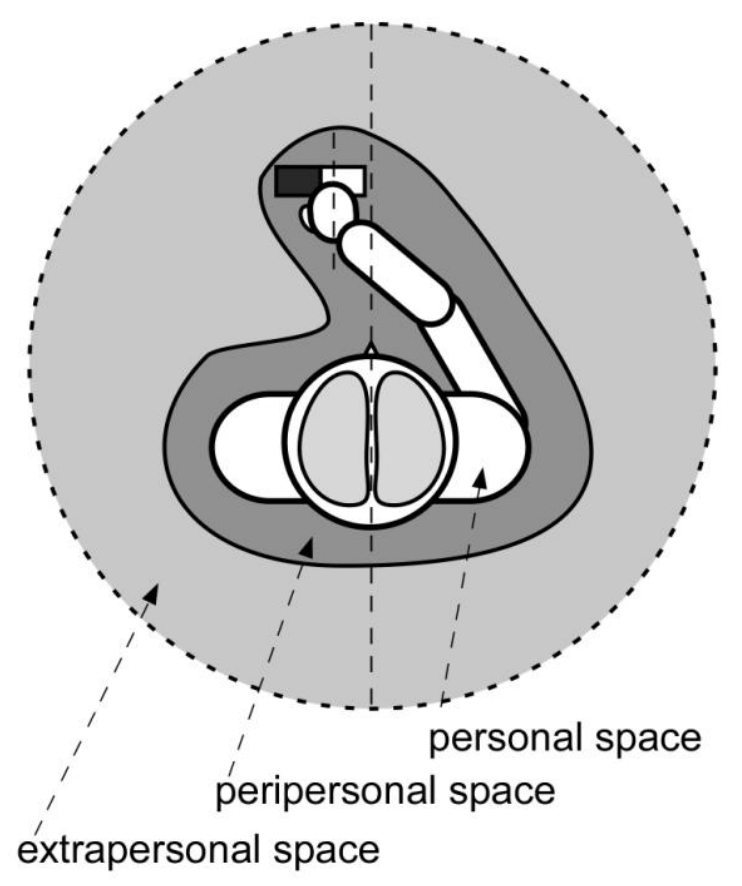

\section{Figure legend}

Fig. 1. Neuropsychologists dissociate usually three main organizations of space: the personal space corresponding to the space of the body, the extrapersonal space in which objects are reached by movements of the limbs, and the intermediate peripersonal space, the space surrounding the body in which objects can be grasped [7]. The peripersonal space is multimodal per essence [8]. A second dissociation can be made between the anatomical frames of reference (e.g., somatotopic, retinotopic) and the spatiotopic frames of reference. For example, in the illustration, tactile information arising from the right hemibody by touching the object with the right hand is sent to the left hemisphere according to the somatotopic frame of reference, but we are able to acknowledge that the object is touching 
the hand in the left side of space from a spatiotopic frame of reference. The object can also be localized on the basis of egocentric (relative to the observer's own body) vs. allocentric (relative to another object) frames of reference. For example, from an object-centered allocentric perspective, the white part of the object is in the right side relative to the midline of the object. In an egocentric perspective, both the black and the white parts of the object are in the left space relative to the midline of the trunk or relative to the midline of the head, but the white part is in the right space relative to the midline of the right hand. The peripersonal space is considered as an interplay of body-part-centered coordinates mapping stimuli from the different senses and moving in space with the body part to which these maps are anchored [8]. 